\title{
Copeptin levels in patients with coronary artery ectasia
}

\author{
OZKAN YAVCIN $^{1}$, LUTFU ASKIN ${ }^{1, *}$, OZLEM SEÇEN ${ }^{2}$, SERDAR TURKMEN ${ }^{1}$, \\ ERDAL AKTURK ${ }^{1}$, OKAN TANRIVERDI ${ }^{1}$, MUSTAFA NECATI DAGLI $^{3}$ \\ ${ }^{1}$ Department of Cardiology, Adiyaman Education and Research Hospital, Adiyaman, Turkey \\ ${ }^{2}$ Department of Cardiology, Elazig Education and Research Hospital, Elazig, Turkey \\ ${ }^{3}$ Department of Cardiology, Elazig Medical Park Hospital, Elazig, Turkey \\ *Corresponding author: Lutfu Askin, MD; Department of Cardiology, Adiyaman Education and Research Hospital, Adiyaman 2230, Turkey; \\ Phone: +90 531 5203486; Fax: +90 4161015; E-mail: lutfuaskin23@gmail.com
}

(Received: February 25, 2019; Revised manuscript received: March 30, 2019; Accepted: April 3, 2019)

\begin{abstract}
Background and aims: The etiology and pathophysiology of coronary artery ectasia (CAE) has not been fully elucidated. A rapid rise in plasma copeptin has been observed in cardiovascular diseases, stroke, sepsis, and shock. This increase has diagnostic and prognostic value. The aim of this study was to investigate whether copeptin has a relationship with CAE. Methods: This observational prospective study was carried out between October 2012 and March 2013 in the cardiology catheter laboratory with the inclusion of 44 subjects with a diagnosis of CAE and 44 age- and gender-matched individuals with normal coronary arteries. Blood samples obtained from the patients were stored at $-70{ }^{\circ} \mathrm{C}$ until analysis and copeptin levels in sera were measured by ELISA. Results: This study comprised 88 study participants, among whom 44 were patients meeting ectasia criteria [mean age: $58.0 \pm 11.5$ years; $59 \%(n=26)$ male], and 44 were control subjects with angiographically normal coronary anatomy [mean age: $49.2 \pm 10.1$ years; $30 \%(n=13)$ male]. Copeptin levels were similar between the groups $(p>0.05)$. In addition, there was no correlation between copeptin levels and CAE. Conclusion: In this study, it is examined that copeptin levels were not elevated in CAE patients.
\end{abstract}

Keywords: cardiovascular diseases, coronary artery ectasia, copeptin level, stroke, sepsis

\section{Introduction}

Coronary artery ectasia (CAE) is defined as a congenital or acquired coronary abnormality involving localized or diffuse enlargement of an epicardial coronary artery to greater than 1.5 times the diameter of an adjacent normal coronary artery [1]. The reported incidence for CAE ranges between $0.3 \%$ and $10 \%[1,3-5]$. Destruction of the muscoelastic elements of the media layer, deposition of collagen and elastin, and the resultant thinning of the vessel wall represent the fundamental processes in the pathogenesis of ectasia $[6,7]$. Injury in the media layer leads to reduced stress tolerance to intraluminal pressure, eventually causing progressive dilatation and ectasia. Simultaneous thinning of the vessel wall and increased wall stress will create a viscous cycle that results in diffuse or local ectasia due to progressive dilatation of the coronary artery [8]. It has been reported that atherosclerosis $(50 \%)$ and inflammatory or connective tissue diseases (20\%-30\%) play an important role in CAE etiology [9].

Copeptin is secreted from the posterior pituitary gland simultaneously with vasopressin, and its levels are indicative of circulating vasopressin. In contrast with arginine vasopressin (AVP), copeptin exhibits higher plasma and serum stability, allowing easy measurement in blood. After release, it remains stable for several days and copeptin levels increase rapidly in conditions, such as cardiovascular disease, stroke, sepsis, and shock; this increase is both indicative of elevated AVP secretion and has diagnostic and prognostic implications [10].

The etiopathogenesis of CAE remains elusive, despite a number of hypotheses proposed. Our objective was to examine the relationship between copeptin levels and CAE.

This is an open-access article distributed under the terms of the Creative Commons Attribution-NonCommercial 4.0 International License, which permits unrestricted use, distribution, and reproduction in any medium for non-commercial purposes, provided the original author and source are credited, a link to the CC License is provided, and changes - if any - are indicated. 


\section{Materials and Methods}

This observational prospective study was undertaken between October 2012 and March 2013 in the cardiology catheter laboratory with the inclusion of 88 subjects with either a diagnosis of CAE or normal coronary anatomy. CAE was defined as a congenital or acquired coronary abnormality involving localized or diffuse enlargement of an epicardial coronary artery to more than 1.5 times the diameter of an adjacent normal coronary artery [4]. We included CAE patients and age- and sexmatched controls.

The exclusion criteria were as follows: presence of kidney failure or impaired renal clearance, congestive heart failure, malignancy, hepatic failure, actual or potential pregnancy, coexistence of coronary lesions associated with $\geq 50 \%$ obstruction, acute myocardial infarction (MI), stroke, severe cardiac valve disease, and previous coronary artery disease (CAD) history.

Coronary angiography procedures were performed using a Philips Allura Xper FD10 device (Philips Medical Systems, Best, The Netherlands) in all patients, with standard $6 \mathrm{~F}$ Judkins right and left catheterization following femoral arterial puncture. Iodixanol or iohexol was used as radiopaque material. Images in least two projections were obtained and recorded in the digital memory and cine-films for the left and right coronary systems, respectively. In patients with suspected ectasia based on visual inspection, quantitative measurements were performed using the measurement software of a Philips Allura Xper FD10 device. In order to ascertain the actual coronary artery lumen width, calibration was performed using the catheter diameter. CAE was defined as localized or diffuse enlargement of an epicardial coronary artery to more than 1.5 times the diameter of an adjacent normal coronary artery. Segments without stenosis and ectasia on angiographic images were considered as normal coronary arterial segments. Body mass index (BMI) was calculated using subject's height and weight. The formula is $\mathrm{BMI}=\mathrm{kg} / \mathrm{m}^{2}$, where $\mathrm{kg}$ is subject's weight in kilogram and $\mathrm{m}^{2}$ is subject's height in meter squared. Left ventricular ejection fraction (LV-EF) was assessed using the modified Simpson's method.

For blood tests, $12 \mathrm{ml}$ of venous blood sample was collected from the left antecubital veins from all patients after overnight fasting. Samples were assessed on the day of sampling using Seimens Advia 1800 auto-analyzer device (XE-5000, Sysmex Corp., Kobe, Japan). Routine biochemical parameters (glucose, lipid profile, and urea creatinine) and complete blood counts (hemoglobin, hematocrit, platelets, and leukocytes) were recorded for each patient. For serum copeptin assays, $3 \mathrm{ml}$ of venous blood was placed into ethylenediaminetetraacetic acid tubes. After the tubes were gently shaken, they were placed in the centrifuge and were centrifuged at $3,500 \mathrm{rpm}$ for $10 \mathrm{~min}$ for the separation of the sera. The resultant samples were maintained at $-70{ }^{\circ} \mathrm{C}$ until the time of measurements. After collection of all samples, serum copeptin levels were measured using "Human Copeptin ELISA Kits" (Phoneix Pharmaceuticals, USA; catalog no.: EK-065-32; lot no.: 603.858)" in microplate readers (Bio-Tek Instruments ELx800, USA) at $450 \mathrm{mg}$ in $\mathrm{pg} / \mathrm{ml}$, in accordance with manufacturer's instructions [11]. The minimum detection concentration, linearity, and the maximum measurement concentration of the kits are $0.12,0.12-1.4$, and $100 \mathrm{ng} / \mathrm{ml}$, respectively. The reported intra-assay [coefficient of variation $(\mathrm{CV})$ : variation within a day] and inter-assay variations (CV: variation between days) for these kits are $<10 \%$ and $<15 \%$, respectively.

\section{Statistical analyses}

Statistical analyses were performed using SPSS 24.0 software package (SPSS Inc., Chicago, IL, USA). The normal distribution of the numerical variables was examined using Kolmogorov-Smirnov test. For copeptin levels, which were not normally distributed, logarithmic transformation was applied prior to statistical analyses. Descriptive statistics are expressed using mean \pm standard deviation for numerical variables, and using number and percentage for categorical variables. Between-group differences for categorical variables were analyzed using $\chi^{2}$ test, whereas independent sample $t$-test was used for the analysis of parametric data analysis. Correlations between variables were assessed using Pearson's and Spearman's correlation coefficient analyses and $p$ values less than 0.05 were considered significant.

\section{Results}

This study comprised 88 study participants, among whom 44 were patients meeting the ectasia criteria [mean age: $58.0 \pm 11.5$ years; $59 \%(n=26)$ male $]$ and 44 were control subjects with angiographically normal coronary anatomy [mean age: $49.2 \pm 10.1$ years; $30 \%(n=13)$ male]. The demographic, clinical, and biochemical assessment characteristics of the patients are shown in Table I. Significant were found between the patient and the control groups in terms of frequencies of CAD risk factors, such as smoking, diabetes, hypertension, and hyperlipidemia $(p<0.05)$. The demographic characteristics such as age and gender were significantly higher in CAE group $(p<0.05)$. The levels of LDL, creatinine, copeptin (Fig. 1), hemoglobin, and plasma glucose were found to be similar between groups $(p>0.05)$. No significant differences were found in terms of BMI, LV-EF, and systolic and diastolic blood pressures $(p>0.05)$.

Spearman's and Pearson's analyses suggested that no such correlation was found between CAE and the factors (Table II). 
Yavcin et al.

Table I Demographic, clinical, and biochemical assessment of study subjects

\begin{tabular}{lccr} 
Baseline variables & Controls $(n=44)$ & Patients with CAE $(n=44)$ & $p$ value \\
Gender [male, $n(\%)]$ & $13(30 \%)$ & $26(59 \%)$ & 0.032 \\
Age (years) & $49.20 \pm 10.15$ & $58.07 \pm 11.53$ & 0.025 \\
Hypertension $[n(\%)]$ & $10(22.7 \%)$ & $20(45.5 \%)$ & 0.007 \\
Smoking $[n(\%)]$ & $12(27.3 \%)$ & $13(29.5 \%)$ & 0.402 \\
Diabetes mellitus $[n(\%)]$ & - & $7(15.9 \%)$ & $<0.001$ \\
BMI $\left(\mathrm{kg} / \mathrm{m}^{2}\right)$ & $26.4 \pm 1.3$ & $27.2 \pm 1.8$ & 0.574 \\
LV-EF $(\%)$ & $58.2 \pm 3.6$ & $57.4 \pm 2.8$ & 0.688 \\
Systolic BP $(\mathrm{mmHg})$ & $115.2 \pm 10.4$ & $117.0 \pm 23.7$ & 0.288 \\
Diastolic BP $(\mathrm{mmHg})$ & $72.8 \pm 9.8$ & $75.0 \pm 9.5$ & 0.156 \\
Plasma glucose $(\mathrm{mg} / \mathrm{dl})$ & $88.7 \pm 21.4$ & $95.7 \pm 29.4$ & 0.188 \\
Hemoglobin $(\mathrm{g} / \mathrm{dl})$ & $13.5 \pm 1.6$ & $14.4 \pm 4.5$ & 0.358 \\
Cre $(\mathrm{mg} / \mathrm{dl})$ & $0.78 \pm 0.17$ & $0.86 \pm 0.21$ & 0.013 \\
LDL $(\mathrm{mg} / \mathrm{dl})$ & $119.0 \pm 33.1$ & $118.7 \pm 41.2$ & 0.369 \\
Copeptin $(\mathrm{p} / \mathrm{mol} / \mathrm{L})$ & $7.9 \pm 1.1$ & $8.2 \pm 0.6$ & 0.222 \\
\hline
\end{tabular}

BMI: body mass index; BP: blood pressure; Cre: creatinine; CAE: coronary artery ectasia; LDL: low-density lipoprotein; LV-EF: left ventricular ejection fraction

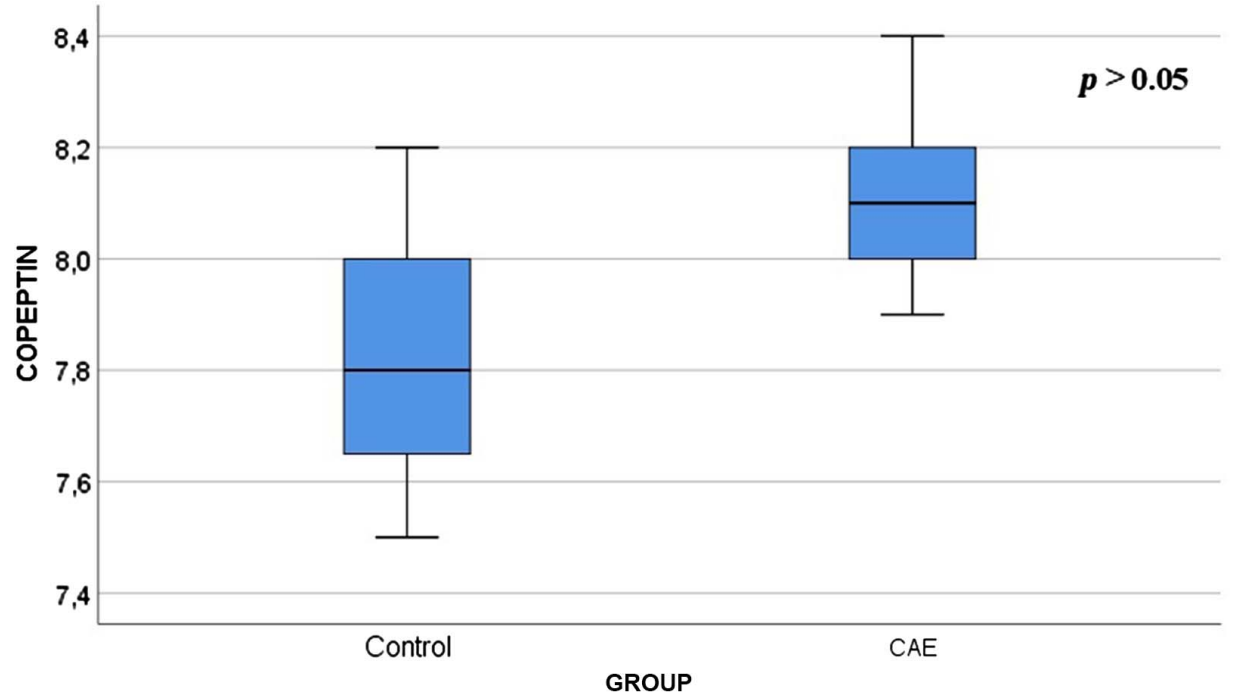

Fig. 1. Serum copeptin levels between the groups

\section{Discussion}

This study demonstrated that serum copeptin levels were similar between CAE patients and controls. No relationship was obvious between CAE and copeptin levels.

The reported incidence of $\mathrm{CAE}$ in various patient series ranges between $0.3 \%$ and $5.3 \%$ [4]. Although CAE is generally without symptoms, it bears clinical significance with regard to its predisposition to cause myocardial ischemia. The etiopathogenesis and pathophysiology of CAE remains obscure, despite many hypotheses that have been put forward.
Despite being a disorder of unknown etiopathogenesis, it is generally regarded as a variant of coronary atherosclerosis. On the other hand, in a significant proportion of patients, CAE is also associated with nonatherosclerotic conditions such as inflammatory diseases, connective tissue disorders, and congenital conditions [12]. Previous studies have reported that NO levels have been shown to have a major role in the development of ectasia and inflammation $[13,14]$.

Copeptin levels rapidly increase in conditions, such as cardiovascular disease, stroke, sepsis, and shock; this increase is indicative of elevated AVP secretion and has 
Table II Factors associated with coronary artery ectasia

\begin{tabular}{lcc} 
& $\begin{array}{c}\text { Univariate analysis } \\
\text { Correlation coefficient }(r)\end{array}$ & $p$ value \\
Age & 0.129 & 0.161 \\
LV-EF & -0.156 & 0.369 \\
Copeptin & 0.208 & 0.102 \\
Plasma glucose & 0.222 & 0.092 \\
LDL & 0.052 & 0.654 \\
BMI & 0.089 & 0.322 \\
\hline
\end{tabular}

BMI: body mass index; LDL: low-density lipoprotein; LV-EF: left ventricular ejection fraction

diagnostic and prognostic implications [10]. Recently, several studies have examined the role of this novel marker in conditions, such as acute MI, chronic obstructive lung disease, sepsis, and shock.

In patients presenting with MI, copeptin has been shown to be a strong predictor of worse outcome and a prognostic marker of death [15]. Of note, its relevance was increased when used in combination with other biomarkers (NT-proBNP and troponins) [16].

Several authors have focused on the diagnostic performance of copeptin in the setting of non-ST-segment elevation of acute coronary syndrome and chest pain. As endogenous stress is increased at the onset of acute coronary syndrome, copeptin could identify acute coronary syndrome patients when other biomarkers are still negative. The use of a "dual-marker" strategy in order to rapidly rule out a diagnosis of acute coronary syndrome in patients presenting with chest pain has been studied in large trials [17].

In a study by Chai et al. [18], significantly higher copeptin levels were found in CAD patients than in controls. Again, plasma copeptin levels exhibited a significant increase 1 day after PTCA or coronary stent placement. Despite a reduction in plasma copeptin levels at 3 and 5 days after the intervention, they remained higher than those in controls.

In a study by Reichlin et al. [19], combined use of plasma copeptin and troponin $\mathrm{T}$ levels was found to have higher sensitivity and specificity as compared to troponin $\mathrm{T}$ alone in ruling out a diagnosis of acute MI.

Copeptin predicts development of CAD and cardiovascular mortality both in diabetic and non-diabetic patients. Copeptin predicts CAD and cardiovascular and total mortality [20,21]. Patients who developed cardiac failure or who died after an acute MI had higher blood copeptin levels in Khan et al.'s [22] study.

In this study, our objective was to explore the relationship between copeptin levels and CAE. To the best of our knowledge, no previous studies have reported such relationship between CAE and copeptin levels.
The copeptin levels play a key role in the diagnosis of acute MI and prognostic significance in patients with low ejection fraction $[23,24]$. However, LV-EF measures were normal and did not differ between groups in this study. CAE frequency is associated with increased risk factors, such as hyperlipidemia, smoking, and hypertension [25-29]. In this study, hyperlipidemia, smoking, and hypertension incidence were similar between CAE patients and controls.

Plasma copeptin levels tend to elevate after stress. A probable explanation for elevated copeptin levels in CAD is the presence of lesions associated with hemodynamically significant narrowing. Thus, absence of significant elevation in copeptin levels in our patient group may be related to the fact that these patients did not have significant narrowing.

\section{Study limitations}

Less number of patients were enrolled in the study because of the rarity of isolated CAE. In addition, subgroup analysis of $\mathrm{CAE}$ was not performed. Another limitation of this study was the lack of evaluation of inflammatory markers, but previous studies have examined the relationship between inflammation and CAE.

\section{Conclusions}

In this study, we found no relationship between copeptin levels and CAE. Further studies to elucidate the pathophysiology of CAE are warranted in order to develop more effective management strategies for this condition.

Funding sources: The funding sponsors had no role in the design of the study; in the collection, analyses, or interpretation of data; in the writing of the manuscript; and in the decision to publish the results.

Authors' contribution: OY, LA, and OS prepared the manuscript. ST gathered data. EA and OT searched the literature. MND and OY analyzed the data. All authors read and approved the final version of the manuscript.

Conflict of interest: The authors declare no conflict of interest regarding the publication of this paper.

\section{References}

1. Yetkin E, Waltenberger J: Novel insights into an old controversy: Is coronary artery ectasia a variant of coronary atherosclerosis? Clin Res Cardiol 96, 331-339 (2007)

2. Markis JE, Joffe CD, Cohn PF, Feen DJ, Herman MV, Gorlin R: Clinical significance of coronary arterial ectasia. Am J Cardiol 37, 217-222 (1976) 
3. Befeler B, Aranda MJ, Embi A, Mullin FL, El-Sherif N, Lazzara R Coronary artery aneurysms: Study of their etiology, clinical course and effect on left ventricular function and prognosis. Am J Med 62, 597-607 (1977)

4. Hartnell GG, Parnell BM, Pridie RB: Coronary artery ectasia. Its prevalence and clinical significance in 4993 patients. Br Heart J 54, 392-395 (1985)

5. Antoniadis AP, Chatzizisis YS, Giannoglou GD: Pathogenetic mechanisms of coronary ectasia. Int J Cardiol 130, 334-335 (2008)

6. Chrissoheris MP, Donohue TJ, Young RS, Ghantous A: Coronary artery aneurysms. Cardiol Rev 16, 116-123 (2008)

7. Jian-Jun L, Zheng L, Jie L: Is any link between inflammation and coronary artery ectasia? Med Hypotheses 69, 678-683 (2007)

8. Gussenhoven EJ, Frietman PA, The SH, van Suylen RJ, van Egmond FC, Lancée CT: Assessment of medial thinning in atherosclerosis by intravascular ultrasound. Am J Cardiol 68, 1625-1632 (1991)

9. Abid AR, Jalala SA, Gad MG, Gehani AA: Clinical and angiographic features of patients with coronary artery ectasia compared with stenotic coronary artery disease. J Cardiol Curr Res 9, 00328 (2017)

10. Morgenthaler NG: Copeptin: A biomarker of cardiovascular and renal function. Congest Heart Fail 16, 37-44 (2010)

11. Ede H, Karaçavuş S, Göçmen AY, Yaylak B, Akkaya S, Açıkgöz B: Serum copeptin level can be a helpful biomarker in evaluation of myocardial perfusion scintigraphy results. Cardiol J 23, 71-77 (2016)

12. Gunes Y, Boztosun B, Yildiz A, Metin Esen A, Saglam M, Bulut M: Clinical profile and outcome of coronary artery ectasia. Heart 92, 1159-1160 (2006)

13. Johanning JM, Franklin DP, Han DC, Carey DJ, Elmore JR: Inhibition of inducible nitric oxide synthase limits nitric oxide production and experimental aneurysm expansion. J Vasc Surg 33, 579-586 (2001)

14. Fukuda S, Hashimoto N, Naritomi H, Nagata I, Nozaki K, Kondo $S$ : Prevention of rat cerebral aneurysm formation by inhibition of nitric oxide synthase. Circulation 101, 2532-2538 (2000)

15. O'Malley RG, Bonaca MP, Scirica BM, Murphy SA, Jarolim P, Sabatine MS: Prognostic performance of multiple biomarkers in patients with non-ST-segment elevation acute coronary syndrome: Analysis from the MERLIN-TIMI 36 trial (Metabolic Efficiency With Ranolazine for Less Ischemia in Non-ST-Elevation Acute Coronary Syndromes-Thrombolysis In Myocardial Infarction). J Am Coll Cardiol 63, 1644-1653 (2014)

16. Lipinski MJ, Baker NC, Escárcega RO, Torguson R, Chen F, Aldous SJ: Comparison of conventional and high-sensitivity troponin in patients with chest pain: A collaborative meta-analysis. Am Heart J 16, 6-16 (2015)
17. Möckel M, Searle J, Hamm C, Slagman A, Blankenberg S, Huber $\mathrm{K}$ : Early discharge using single cardiac troponin and copeptin testing in patients with suspected acute coronary syndrome (ACS): A randomized, controlled clinical process study. Eur Heart J 36, 369-376 (2015)

18. Chai SB, Hui YM, Li XM, Xiao Y, Tang CS: Plasma levels of copeptin in patients with coronary heart disease. Heart Vessels 24, 79-83 (2009)

19. Reichlin T, Hochholzer W, Stelzig C, Laule K, Freidank H, Morgenthaler NG: Incremental value of copeptin for rapid rule out of acute myocardial infarction. J Am Coll Card 54, 60-68 (2009)

20. Tasevska I, Enhörning S, Persson M, Nilsson PM, Melander O: Copeptin predicts coronary artery disease cardiovascular and total mortality. Heart 102, 127-132 (2016)

21. Enhörning S, Hedblad B, Nilsson PM, Engström G, Melander O: Copeptin is an independent predictor of diabetic heart disease and death. Am Heart J 169, 549-556 (2015)

22. Khan SQ, Dhillon OS, O'Brien RJ, Struck J, Quinn PA, Morgenthaler NG: C-terminal provazopressin (copeptin) as a novel and prognostic marker in acute myocardial infarction. Circulation $115,2103-2110$ (2007)

23. Möckel M, Searle J: Copeptin-marker of acute myocardial infarction. Curr Atheroscler Rep 16, 421 (2014)

24. Pozsonyi Z, Förhécz Z, Gombos T, Karádi I, Jánoskuti L, Prohászka Z: Copeptin (C-terminal pro arginine-vasopressin) is an independent long-term prognostic marker in heart failure with reduced ejection fraction. Heart Lung Circ 24, 359-367 (2015)

25. Pinar Bermúdez E, López Palop R, Lozano Martínez-Luengas I, Cortés Sánchez R, Carrillo Sáez P, Rodríguez C: Coronary ectasia: Prevalence, and clinical and angiographic characteristics. Rev Esp Cardiol 56, 473-479 (2003)

26. Kruger D, Stierle U, Herrmann G, Simon R, Sheikhzadeh A: Exercise-induced myocardial ischemia in isolated coronary artery ectasias and aneurysms ("dilated coronopathy"). J Am Coll Cardiol 34, 1461-1470 (1999)

27. Adiloglu AK, Can R, Nazli C, Ocal A, Ergene O, Tinaz G: Ectasia and severe atherosclerosis: Relationships with chlamydia pneumoniae, helicobacter pylori, and inflammatory markers. Tex Heart Inst J 32, 21-27 (2005)

28. Yilmaz H, Tayyareci G, Sayar N, Gurkan U, Tangurek B, Asilturk $\mathrm{R}$ : Plasma soluble adhesion molecule levels in coronary artery ectasia. Cardiology 105, 176-181 (2006)

29. Von Haehling S, Papassotiriou J, Morgenthaler NG, Hartmann O, Doehner W, Stellos K: Copeptin as a prognostic factor for major adverse cardiovascular events in patients with coronary artery disease. Int J Cardiol 162, 27-32 (2012) 